\title{
Metabolomics in experimental neonatology
}

\begin{abstract}
Metabolomics is a new 'omics' science, based on the thorough understanding of the metabolic fingerprint, thus recording the so called 'metabolic signature' of host's cellular response. Recent data highlight that novel metabolic biomarkers reflect the multiple network interactions taking part in the host's response to the disease burden, treatment intervention or life threatening conditions. Therefore, fetal or neonatal metabolome might be an early predictor of the ongoing metabolic procedures or molecular changes, thus a promising tool for early detection and prompt intervention. Experimental protocols on newborn animals have a significant role on lightening this foggy landscape.
\end{abstract}

Keywords: metabolomics, neonatology, experimental
Volume 2 Issue 4 - 2015

\author{
Afroditi Aggelina,' Angeliki Syggelou,' \\ Athanasios Chalkias, 'Apostolos Papalois, ${ }^{2}$ \\ Theodoros Xanthos,' Nicoletta lacovidou ${ }^{3}$ \\ 'National and Kapodistrian University of Athens, Greece \\ ${ }^{2}$ ELPEN Research-Experimental Centre, Greece \\ ${ }^{3}$ Neonatal Department, National and Kapodistrian University of \\ Athens, Greece
}

Correspondence: Nicoletta lacovidou, Neonatal Department, National and Kapodistrian University of Athens, Medical School, Pavlou Mela, 16233 Athens, Greece, Email niciac85@gmail.com

Received: July 10, 2015 | Published: August 28, 2015
Abbreviations: LPS, lipopolysaccharide; OGD, oxygenglucose deprivation; ATP, adenosine triphosphate; NMR, nuclear magnetic resonance; BAL, bronchoalveolar lavage; TCA, tricarboxylic acid; IUGR, intrauterine growth restriction; ALI, acute lung injury

\section{Introduction}

Metabolomics is a new 'omics' discipline, based on the detailed analysis of the host's metabolic phenotype and the complex metabolic pathways with multiple network interactions. This metabolic fingerprint is considered as the gold standard for the profound understanding of the normal metabolic processes and of the alterations of the normal pathways. This 'snapshot' of the metabolic status may be a reliable tool for the prediction, early detection and monitoring the disease progression and may allow the identification of innovative interventions for delaying or reversing abnormal molecular changes. Non-human models have been used for the analysis of the metabolic status of the fetus or neonate in certain clinical conditions and existing data allow a more open-minded approach of studying and interpreting the fetal or neonatal metabolome. Therefore, metabolomics in experimental neonatology have gained a large impact in life sciences over the last decade.

\section{Discussion}

Non-human models have been used extensively for metabolomics analysis and various experimental protocols in metabolomics research have been published in literature Table 1. In a newborn piglet model of hypoxia, authors successfully identified 13 urinary metabolites that differentiated hypoxic versus nonhypoxic animals. These metabolites were 1-methylnicotinamide, 2-oxoglutarate, alanine, asparagine, betaine, citrate, creatine, fumarate, hippurate, lactate, Nacetylglycine, $\mathrm{N}$-carbamoyl-b-alanine, and valine, all directly related to cellular energy levels and metabolism. This metabolomic profile was able to blindly identify hypoxic and non-hypoxic animal's correctly. ${ }^{1}$

Metabolic studies including hypoxia and reoxygenation with different oxygen concentrations in newborn piglets highlighted strong variations in plasma metabolic parameters. Three groups of hypoxic newborn piglets received respectively $100 \%$ oxygen for $60 \mathrm{~min}$, $21 \%$ oxygen for $60 \mathrm{~min}, 100 \%$ for $15 \mathrm{~min}$ and then $21 \%$ oxygen for $45 \mathrm{~min}$. In the hypoxic phase, lactate, low $\mathrm{PH}$ and base deficit were independent of the duration of hypoxia, while prolonged hypoxia led to low levels of free and total carnitine and an increase in long chain acyl-carnitines, which have toxic effect. In the reoxygenation phase, the subsequent hyperoxia was associated with a slower decline of Krebs cycle intermediates and an increase in lanosterol and in oxysteroles, both indicative of acute neuronal damage. ${ }^{2}$

Fetal sheep and its plasma metabolome following inflammatory stimuli with E.coli lipopolysaccharide (LPS) were analyzed by Keller et al. ${ }^{3}$ Induced hypoxia via LPS injection to the umbilical vein, resulted in various metabolic alterations. Krebs cycle intermediates, alanine and lactate, spermidine and oxysteroles with a known pro-apoptotic effect, were early increased while a decrease in hexoses was reported. Days after the hypoxic insult, there was a delayed opposite effect with remarkable hyperoxia, elevation of mediators of inflammation such as sphingomyelins, kynurenine, 3-hydroxykynurenine, putrescine, asymmetric dimethyl arginine, the latter well known for its role in microvascular tone and endothelial function.

A similar non-human model experiment was described by Atzori and his co-workers; normocapnic hypoxia was induced in 4 groups of newborn piglets to either bradycardia or severe hypotension and different oxygen concentrations were used for resuscitation; urine samples were collected for metabolomics analysis. Despite reoxygenation 7 piglets out of the 10 piglets which became asystolic during the experiment, died. Urine analysis revealed differences in the metabolic profile of the urine collected before the induction of hypoxia between survivors and deaths; these included methyl guanidine and hydroxyisobutyric acid, malonate, urea, and creatinine, the three latter ones being well known for their role in aerobic metabolism, neurological and renal disorders as well as cellular death. These findings may establish new strategies in oxygen administration interventions, thus avoiding mortality related to hyperoxia. ${ }^{4}$

Beckstroma and Humstonb worked on an experimental model of perinatal asphyxia in Macaca nemestrina newborns. Umbilical cord occlusion following hysterectomy was performed and blood samples were collected before and 15 or 18 minutes after the hypoxic stimuli as well as 5 minutes postnatal. Authors reported a great variation in metabolite levels pre- to post- asphyxia, 10 of which remained significantly different compared to control animals. Specifically, lactate and creatinine were strongly correlated to hypoxic-ischemic injury due to perinatal asphyxia and an increase in the Krebs cycle's intermediates and arachidonic acid was indicative of the complex alterations in metabolic pathways. ${ }^{5}$ 
Table I Summary of neonatal experimental protocols on metabolomics, LPS (lipopolysaccharide), BAL (bronchoalveolar lavage), and IUGR (intrauterine growth restriction)

\begin{tabular}{|c|c|c|c|}
\hline Experimental Protocol & Animals & Sample & Omics Analysis \\
\hline \multirow[t]{6}{*}{ Asphyxia } & Piglets & Blood & Increased ratio of Alanine, \\
\hline & & & Glycine/Branched-chain amino acid \\
\hline & & Urine & increased creatinine, urea, malonate, \\
\hline & & & uric acid, methylguanidine \\
\hline & Rats & Heart plasma & reduced plasma/cardiac concentrations \\
\hline & & & of short chain acylcarnitines \\
\hline IUGR & Rats & Plasma & fatty acid alterations in long term \\
\hline \multirow[t]{2}{*}{ Sepsis } & Rats & Serum, BAL, & alterations in alanine, formate, creatine, \\
\hline & & Lung Tissue & phosphoethanolamine \\
\hline \multirow[t]{3}{*}{ Thermal Injury/sepsis } & Rats & Serum & alterations in hypoxanthine, indoxyl \\
\hline & & & sulfate, glutamic acid, proline, uracil, \\
\hline & & & uric acid, trihydroxy cholanic acid \\
\hline \multirow[t]{2}{*}{ Brain Injury after LPS } & Sheeps & Brain & increased Krebs cycle intermediates, \\
\hline & & & oxysteroles, spermidine \\
\hline Gentamycine Induced & Rats & Urine & increased glucose, $\mathrm{N}$-acetylglucosamine, \\
\hline Nephrotoxicity & & & myo-inositol, butanoic acid, 3 hydroxybutirate \\
\hline
\end{tabular}

Furthermore, a hypothermia protocol highlighted the metabolic alterations related to neonatal brain injury. Three different experimental procedures were performed, including $45 \mathrm{~min}$ oxygen-glucose deprivation (OGD) with a 3-hour mild hypothermia $\left(32^{\circ} \mathrm{C}\right)$, OGD followed by hypothermia after a 15 -min delay and total normothermia $\left(37^{\circ} \mathrm{C}\right)$ in the control group while in all groups hypothermia was followed by a 3-hour normothermic recovery. The metabolic snapshot revealed early restoration of the initial adenosine triphosphate (ATP) levels in the 2 hypothermia treated groups, whereas cellular death was greater with normothermia and delayed hypothermia, compared to immediate hypothermia. This 'omics' analysis indicated the complex cellular function; therefore more data is needed to shed light on optimal cooling and rewarming regimens. ${ }^{6}$

Nuclear magnetic resonance (NMR)-based metabolomics analysis was performed in a model of cecal ligation and puncture procedure in rats, which is the gold standard method to investigate the clinical settings of sepsis and septic shock. ${ }^{7}$ Bronchoalveolar lavage (BAL) fluid, lung tissue and serum samples were obtained from septic rats and the control group as well. Multiple metabolic signaling pathways involved in inflammatory process and sepsis-induced cell damage were highly discriminated. Alanine concentration in lung tissue and serum samples of septic rats, derived from enhanced pyruvate metabolism and transamination to alanine via the Cori cycle, was interestingly increased. ${ }^{8}$ Creatine, a nitrogenous organic acid which was found in high levels in lung tissue, BAL as well as in serum ${ }^{9}$ and the high phosphoethanolamine and acetoacetate in the serum of septic rats may easily indicate septic stimuli and ongoing cell damage. ${ }^{10,11}$ Finally, the high levels of myo-inositol found in lung tissue, but in low concentrations in BAL, indicated an altered role in endotoxin-induced vascular smooth muscle hypocontractility. ${ }^{12,13}$

Similarily, post term primates gave birth by hysterotomy and prebirth blood samples followed by post-birth samples at 8 time-points were collected and analyzed. The levels of the Tricarboxylic Acid (TCA) cycle intermediates ( $\alpha$-ketoglutaric acid, fumaric acid, malic acid, and succinyl-CoA), myo-inositol and glutaminic acid were high in serum samples. These findings suggested the metabolic alterations related to birth transition, while myo-inositol and glutaminic acid signaled molecules in adverse metabolic pathways resulting in hypoxic ischemic encephalopathy. ${ }^{14}$
In two experimental studies in newborn rats, urine metabolome in a gentamicin induced nephrotoxicity protocol was analyzed. Various parameters, such as creatine, nicotinic acid, prostaglandin E2 and cholic acid were identified as phenotypic biomarkers of gentamicininduced toxicity. Moreover, authors declared that specific metabolites, namely glucose, galactose, $\mathrm{N}$-acetylglucosamine, myo-inositol, butanoic acid and 3 hydroxybutirate, were significantly increased while citruline, pseudouridine were detected in low levels. ${ }^{15-17}$ Detailed lipid profile analysis was performed in neonatal and young adult rats after a 2-hourly hypoxia and recovery phase. Hypoxic neonates had decreased levels of cardiac and plasma concentrations of short-chain acylcarnitines as well as most plasma long-chain fatty acids. On the contrary, recovery from hypoxia resulted in increased concentrations of propionylcarnitine, palmitoylcarnitine, stearoylcarnitin in neonatal hearts, while oleylcarnitine and linoleylcarnitine concentrations were increased in neonatal plasma. The concentrations of long-chain fatty acids and long-chain acylcarnitines were increased in the hearts and plasma of hypoxic young adult rats; this lipid profile analysis highlighted the great metabolic and physiologic flexibility of neonatal heart and plasma after hypoxia, which increases chances for survival. ${ }^{18}$

Alexandre-Gouabau et al. ${ }^{19}$ reported that the metabolome and the postnatal growth velocity of newborn rats with intrauterine growth restriction (IUGR) were strongly related to metabolic diseases and obesity in adulthood. Offspring's were divided in 3 groups, those exposed to maternal adequate protein diet during pregnancy and lactation (CC) or protein deprivation during pregnancy only (IUGR with rapid catch-up growth, RC) or through pregnancy and lactation (IUGR with slow postnatal growth, RR) and their plasma metabolome was analyzed in early life (until weaning) and once they reached adulthood (8 months). In particular, transient alterations in proline, arginine, and histidine in RR rats, compared to $\mathrm{CC}$ rats, were observed and persistent differences in tyrosine and carnitine, compared to RC rats in adulthood were also highlighted.

Acute lung injury (ALI) was an interesting field where metabolomics were also applied. Increased mortality from ALI was strongly related to cell-specific biomarkers including specific cytokines and their receptors (IL-6, IL-8, soluble tumor necrosis factor receptor I and II), products of epithelial and endothelial injury such as receptor for advanced glycation end-products, surfactant protein D, von Willebrand factor antigen as well as coagulation factors (protein 
$\mathrm{C}$ and plasminogen activator inhibitor-1). Moreover, in sepsisinduced ALI, preliminary data suggested a positive correlation with individual metabolites including myo-inositol and total glutathione. These findings provide the first insight into the metabolic profiles of plasma samples from patients with ALI. ${ }^{20}$

The application of metabolomics in pharmaceutical research of drug candidates and drug safety evaluation may identify drugrelated alterations in metabolic pathways. In an experimental protocol including preterm pigs, control treatment or broad-spectrum antibiotics were given just after delivery by cesarean section. Authors declared that urine metabolome is a promising tool as an early biomarker of NEC progression in preterm neonates who received antibiotics. ${ }^{21}$ Moreover, data analyzed the changes in metabolic profiles of urine and fecal extracts of 15 mice following gut sterilization by the broad-spectrum antibiotic enrofloxacin (also known as Baytril). Urine metabolome analysis revealed that enrofloxacin treatment was associated with decreased acetate due to loss of microbial catabolism of sugars and polysaccharides, decreased trimethylamine- $\mathrm{N}$-oxide due to loss of microbial catabolism of choline, and increased creatine and creatinine due to loss of microbial enzyme degradation. Similarily, in fecal extracts reduction in amino acids produced by microbial proteases was observed, while loss of microbial ureases resulted in increase in urea. These findings suggested that metabolomics might serve as a monitoring tool of the imbalance of the healthy gut flora caused by antibiotic treatment. ${ }^{22}$

Recent data highlight the role of 'omics' science in the analysis of retina samples obtained from asphyxiated and control newborn piglets. This metabolic fingerprint allowed the selection of eight differentiating metabolites. In particular, one of the identified differentiating metabolites, cytidine diphosphate-choline and two related metabolites, choline and acetylcholine were strongly related to the asphyxiated retina, well known as an oxygen-sensitive tissue. As cytidine diphosphate-choline plays a key role in phospholipid synthesis and neuroprotective function under hypoxic conditions, it remains a promising tool for assessing retinal hypoxia. ${ }^{23}$

A major research issue is the role of metabolomics in resuscitation and the thorough understanding of metabolic patterns in perinatal asphyxia. A recent study investigated the urinary metabolic profile of four groups of newborn piglets receiving cardiopulmonary resuscitation and reoxygenation with different oxygen concentrations $(18 \%, 21 \%, 40 \%$, and $100 \%$ respectively) after induced hypoxia and arrest. A baseline urine sample was obtained before hypoxia and a second sample was collected once the animals were reoxygenated and stabilized for 30 minutes. The analysis of the urine metabolites characteristic of each group showed that resuscitation performed with lower oxygen concentration was associated with better cellular homeostasis, cellular maintenance, and carbohydrates metabolisms. The metabolic fingerprint associated with this favorable outcome was related to glucose, lactate, alanine, glyceric acid, pyruvic acid, malonic acid, glycine, succinate, 3-methyladenine, acetylglycine, glutaconic acid, 4-hydroxy-phenyl pyruvic acid, and 3-hydroxymethyl glutarate. On the other hand, resuscitation with higher oxygen concentration was characterized by the presence of creatinine, urea, citric acid, tartaric acid, ethanol, glucose, and indoxyl sulfate. ${ }^{24}$

\section{Conclusion}

Metabolomics has become a rapidly growing area of Systems Biology and it remains a challenge to have reliable biomarkers reflecting the current pathophysiology and easy to determine.
Experimental protocols in newborn animals have been extensively used in order to have a thorough insight into the medical conditions that affect neonates and future perspective is the clinical application of these data in neonatology.

\section{Acknowledgments}

None.

\section{Conflicts on interest}

The author declares that there is no conflict of interest.

\section{Funding}

None.

\section{References}

1. Skappak C, Regush S, Cheung PY, et al. Identifying hypoxia in a newborn piglet model using urinary NMR metabolomic profiling. PLoS One. 2013;8(5):e65035.

2. Solberg R, Enot D, Deigner HP, et al. Metabolomic analyses of plasma reveals new insights into asphyxia and resuscitation in pigs. PLoS One. 2010;5(3):e9606.

3. Keller M, Enot DP, Hodson MP, et al. Inflammatory-induced hibernation in the fetus: priming of fetal sheep metabolism correlates with developmental brain injury. PLoS One. 2011;6(12):e29503.

4. Atzori L, Xanthos T, Barberini L, et al. A metabolomic approach in an experimental model of hypoxia-reoxygenation in newborn piglets: urine predicts outcome. J Matern Fetal Neonatal Med. 2010;3:134-137.

5. Beckstrom AC, Humston EM, Snyder LR, et al. Application of comprehensive two-dimensional gas chromatography with time-offlight mass spectrometry method to identify potential biomarkers of perinatal asphyxia in a non-human primate model. $J$ Chromatogr A. 2011;1218(14):1899-1906.

6. Liu J, Litt L, Segal MR, et al. Outcome-related metabolomic patterns from 1H/31P NMR after mild hypothermia treatments of oxygenglucose deprivation in a neonatal brain slice model of asphyxia. $J$ Cereb Blood Flow Metab. 2011;31(2):547-559.

7. Dejager L, Pinheiro I, Dejonckheere E, et al. Cecal ligation and puncture: the gold standard model for polymicrobial sepsis?. Trends Microbiol. 2011;19(4):198-208.

8. Gore DC, Jahoor F, Hibbert J, et al. Except for alanine, muscle protein catabolism is not influenced by alterations in glucose metabolism during sepsis. Arch Surg. 1995;130(11):1171-1176.

9. Bolton CF. Sepsis and the systemic inflammatory response syndrome: neuromuscular manifestations. Crit Care Med. 1996;24(8):1408-1416.

10. Vulimiri SV, Misra M, Hamm JT, et al. Effects of mainstream cigarette smoke on the global metabolome of human lung epithelial cells. Chem Res Toxicol. 2009;22(3):492-503.

11. Singer M, De Santis V, Vitale D, et al. Multiorgan failure is an adaptive, endocrine-mediated, metabolic response to overwhelming systemic inflammation. Lancet. 2004;364(9433):545-548.

12. Sotoda Y, Negoro M, Wakabayashi I. Involvement of decreased myo-inositol transport in lipopolysaccharide-induced depression of phosphoinositide hydrolysis in vascular smooth muscle. FEBS Lett. 2002;519(1-3):227-230

13. Izquierdo-García JL, Nin N, Ruíz-Cabello J, et al. A metabolomic approach for diagnosis of experimental sepsis. Intensive Care Med. 2011 . 
14. Beckstrom AC, Tanya P, Humston EM, et al. The perinatal transition of the circulating metabolome in a nonhuman primate. Pediatr Res. 2012;71(4 Pt 1):338-344.

15. Fanos V, Antonucci R, Barberini L, et al. Clinical application of metabolomics in neonatology. J Matern Fetal Neonatal Med. 2012;25 Suppl 1:104-109.

16. Syggelou A, Iacovidou N, Atzori L, et al. Metabolomics in the developing human being. Pediatr Clin North Am. 2012;59(5):1039-1058.

17. Hanna M. Metabolomics markers of nephrotoxicity in newborn rats. Poster presented at PAS/ASPR, Denver, US. 2011.

18. Bruder ED, Raff H. Cardiac and plasma lipid profiles in response to acute hypoxia in neonatal and young adult rats. Lipids Health Dis. 2010;9:3.

19. Alexandre-Gouabau MC, Courant F, Le Gall G, et al. Offspring metabolomics response to maternal protein restriction in a rat model of intrauterine growth restriction (IUGR). J Proteome Res. 2011;10(7):3292-3302.
20. Lacy P. Metabolomics of sepsis-induced acute lung injury: a new approach for biomarkers. Am J Physiol Lung Cell Mol Physiol. 2011;300(1):L1-L3

21. Jiang P. Urine metabolome of preterm neonates with treatment of antibiotics. Metabomeeting, Helsinki, Finland. 2011.

22. Romick-Rosendale LE, Goodpaster AM, Hanwright PJ, et al. NMRbased metabonomics analysis of mouse urine and fecal extracts following oral treatment with the broad-spectrum antibiotic enrofloxacin (Baytril). Magn Reson Chem. 2009;47:S36-S46.

23. Solberg R, Escobar J, Arduini A, et al. Metabolomic analysis of the effect of postnatal hypoxia on the retina in a newly born piglet model. PLoS One. 2013;8(6):e6654.

24. Fanos V, Noto A, Xanthos T, et al. Metabolomics network characterization of resuscitation after normocapnic hypoxia in a newborn piglet model supports the hypothesis that room air is better. Biomed Res Int. 2014(2014):7. 